\title{
Adaptive broadband beamforming with arbitrary array geometry
}

\author{
A Alzin*, FK Coutts*, J Corr*, S Weiss*, IK Proudler ${ }^{\dagger}$, JA Chambers ${ }^{\ddagger}$ \\ ${ }^{*}$ Department of Electronic and Electrical Engineering, University of Strathclyde, Glasgow, Scotland \\ ${ }^{\dagger}$ School of Electronic, Electrical and Systems Engineering, Loughborough University, Loughborough, UK \\ ${ }^{\ddagger}$ School of Electrical and Electronic Engineering, Newcastle University, Newcastle upon Tyne, UK \\ \{ahmed.alzin,fraser.coutts,jamie.corr,stephan.weiss\}@strath.ac.uk
}

Keywords: broadband array processing; minimum variance distortionless response beamforming; polynomial matrix; polynomial eigenvalue decomposition.

\begin{abstract}
This paper expands on a recent polynomial matrix formulation for a minimum variance distortionless response (MVDR) broadband beamformer. Within the polynomial matrix framework, this beamformer is a straightforward extension from the narrowband case, and offers advantages in terms of complexity and robustness particularly for off-broadside constraints. Here, we focus on arbitrary 3-dimensional array configurations of no particular structure, where the straightforward formulation and incorporation of constraints is demonstrated in simulations, and the beamformer accurately maintains its look direction while nulling out interferers.
\end{abstract}

\section{Introduction}

Narrowband beamforming has been very extensively addressed over the past three decades, and many robust algorithms exist that offer optimality in various senses. When dealing with broadband signals, time delays cannot be implemented by phase shifts but require explicit tap delay line structures. With this, a number of narrowband techniques such as minimum variance distortionless response (MVDR) beamforming have been extended to the broadband case [21].

While for narrowband arrays, the array configuration can be arbitrary, broadband arrays often are restricted to linear or planar structures, where the look direction of the array is towards broadside. This simplifies the formulation and incorporation of constraints through techniques such as cascades sums of differencing [9] but is practically very restrictive. As a result, most broadband beamformer designs assume presteering i.e. an external provision of time delays - for the look direction to appear at broadside $[19,24,15]$. Alternatively, the beam response can be tied down by a number of frequency domain constraints [16], which however does not guarantee a coherent protection of the constraint in between frequency bins as demonstrated in [23].

In this paper, we employ the recently proposed polynomial matrix formulation in order to denote and solve a broadband MVDR beamformer [23]. The formulation is based on a space-time covariance matrix which explicitly incorporates time lags, and a broadband steering vector. Since every element of such a matrix or vector now is a finite impulse response filter, their transfer functions are polynomial matrices. Narrowband techniques such as eigenvalue (EVD) and singular value decomposition (SVD) have been extended to cover the polynomial matrix case $[6,20,10]$. Algorithms for a polynomial EVD $[10,13,14]$ have been driving forward applications in denoising-type [12] or decorrelating preprocessors [7] for beamforming, transmit and receive beamforming across broadband MIMO channels [4,18], broadband angle of arrival estimation [2], and for optimum subband partitioning of beamformers [22].

The specific aim of this paper is to demonstrate the constraint design and broadband beamformer implementation for an array with arbitrary configuration. The array element locations have to be known, but the elements themselves can be randomly distributed as long as the spatial sampling theorem is satisfied. We extend the definition of a broadband steering vector and metrics such as the directivity pattern to three dimensions, and show how this can be applied in a three dimensional example scenario.

The paper is organised as follows. Sec. 2 will define the array setup, Sec. 3 details the broadband beamformer. The implementation of broadband steering vectors and the blocking matrix required for a generalised sidelobe canceller implementation of the MVDR is addressed in Sec. 4. Finally, Sec. 5 presents some simulation results, followed by conclusions in Sec. 6.

\section{Array Setup and Broadband Steering Vectors}

\subsection{Array Elements}

We assume that an array consists of $M$ elements. There is no assumptions w.r.t. any particular structure of the array, but its element locations are known with Cartesian coordinates $\mathbf{r}_{m}=$ $\left[x_{m}, y_{m}, z_{m}\right]^{\mathrm{T}}, m=1 \ldots M$. The locations may also be given in spherical coordinates with elevation $\vartheta$, azimuth $\varphi$ and radius $\rho$. In this case, a coordinate $\mathbf{r}_{m}$ can also be expressed as

$$
\mathbf{r}_{m}=\rho_{m}\left[\begin{array}{c}
\sin \left(\vartheta_{m}\right) \cos \left(\varphi_{m}\right) \\
\sin \left(\vartheta_{m}\right) \sin \left(\varphi_{m}\right) \\
\cos \left(\vartheta_{m}\right)
\end{array}\right]
$$




\subsection{Far Field Source}

A far-field source has a planar wavefront travelling across the array, arriving at different array elements with different time delays. The normal vector of this wavefront is

$$
\mathbf{k}_{\vartheta, \varphi}=\left[\begin{array}{c}
\sin \vartheta \cos \varphi \\
\sin \vartheta \sin \varphi \\
\cos \vartheta
\end{array}\right]
$$

If normalised by the propagation speed $c$ in the medium, $\mathbf{k}_{\vartheta, \varphi} / c$ is also known as the source's slowness vector. The time delay which the wavefront experiences at the $m$-th element relative to the origin is $t_{m}=\frac{1}{c} \mathbf{k}_{\vartheta, \varphi}^{\mathrm{T}} \mathbf{r}_{m}$. If the unit length in the Cartesian coordinate system is chosen as half the minimum wavelength, then $\left|\mathbf{k}_{\vartheta, \varphi}\right|=1=\lambda_{\min } / 2=c /\left(2 f_{\max }\right)=c T_{\mathrm{s}}$, where $T_{\mathrm{s}}$ is the sampling period assuming critical sampling. Therefore, $t_{m}=\tau_{m} T_{\mathrm{s}}$ with

$$
\tau_{m}=\mathbf{k}_{\vartheta, \varphi}^{\mathrm{T}} \mathbf{r}_{m}
$$

is the wavefront's delay relative to the origin in samples.

\subsection{Broadband Steering Vector}

If at least two array elements are spaced no further than one unit length apart, then a source with direction $\mathbf{k}_{\vartheta, \varphi}$ is uniquely represented by the $M$ delays that its wavefront experiences. This leads to the definition a broadband steering vector, or rather a vector valued function,

$$
\mathbf{s}_{\vartheta, \varphi}[n]=\frac{1}{\sqrt{M}}\left[\begin{array}{c}
f\left[n-\tau_{1}\right] \\
f\left[n-\tau_{2}\right] \\
\vdots \\
f\left[n-\tau_{M}\right]
\end{array}\right],
$$

where $f[n-\tau]$ is an ideal fractional delay filter creating a delay of $\tau \in \mathbb{R}$ samples, with $n \in \mathbb{Z}$ the discrete time index. The lag values $\tau_{m}$ on the r.h.s. of (4) depend on the elevation $\vartheta$ and azimuth $\varphi$ of the source via (3), which is reflected by the subscript of the broadband steering vector, $\mathbf{s}_{\vartheta, \varphi}[n]$. Attenuation of the wavefront while travelling across the array is neglected.

The $z$-transform of $\mathbf{s}_{\vartheta, \varphi}[n]$,

$$
\mathbf{s}(\vartheta, \varphi, z)=\sum_{n=-\infty}^{\infty} \mathbf{s}_{\vartheta, \varphi}[n] z^{-n}
$$

leads to the narrowband steering vector at frequency $\Omega_{0}$ when evaluated on the unit circle for $z=e^{j \Omega_{0}}$. A transform pair as defined in (5) will be abbreviated below as $\mathbf{s}(\vartheta, \varphi, z) \bullet \mathbf{s}_{\vartheta, \varphi}[n]$. Later, the broadband steering vector in (5) will be used to formulate both the broadband MVDR beamforming problem and also its proposed broadband solution.

\section{Broadband Beamformer}

\subsection{General Broadband Beamformer}

In a broadband beamformer, every sensor is followed by a tap delay line as shown in Fig. 1, such that the beamformer output

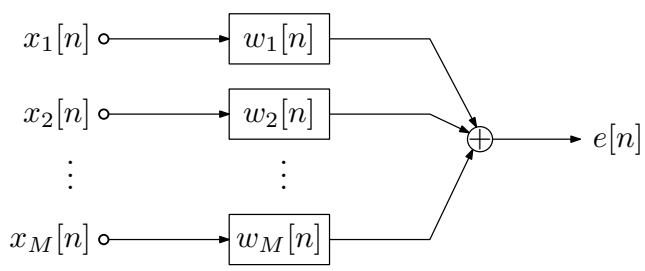

Figure 1. Beamformer with FIR filters $w_{m}[n], m=1 \ldots M$, following each of the $M$ array elements.

$e[n]$ is a weighted average across a spatial and temporal window of the data. If the input vector is defined as

$$
\mathbf{x}[n]=\left[\begin{array}{llll}
x_{1}[n] & x_{2}[n] & \ldots & x_{M}[n]
\end{array}\right]^{\mathrm{T}}
$$

and $\mathbf{w}[n]$ is a vector containing the $M$ impulse responses of the beamforming filters,

$$
\mathbf{w}^{\mathrm{H}}[n]=\left[\begin{array}{llll}
w_{1}[n] & w_{2}[n] & \ldots & \left.w_{M}[n]\right],
\end{array}\right.
$$

then the output is

$$
e[n]=\sum_{\nu} \mathbf{w}^{\mathrm{H}}[\nu] \mathbf{x}[n-\nu]
$$

\subsection{Space-Time Covariance Matrix}

The design of the MVDR beamformer as a statistically optimum array processor [21,5] requires the covariance matrix of the input. Here we choose a representation $\mathbf{R}[\tau]=\mathcal{E}\left\{\mathbf{x}[n] \mathbf{x}^{\mathrm{H}}[n-\tau]\right\}$, which explicitly embeds the lag value $\tau$; this lag can help express temporal correlations arising from time delay in the broadband array data.

The cross-spectral density (CSD) matrix $\boldsymbol{R}(z) \bullet \mathbf{R}[\tau]$ is a polynomial matrix, containing cross- and power spectral densities as elements. Due to the structure of $\mathbf{R}[\tau], \boldsymbol{R}(z)$ is parahermitian, i.e. it is invariant under a parahermitian operation, $\{\tilde{\cdot}\}$, implying Hermitian transpose and time reversal such that $\tilde{\boldsymbol{R}}(z)=\boldsymbol{R}^{\mathrm{H}}\left(z^{-1}\right)=\boldsymbol{R}(z)$.

If the array is illuminated by $L$ sources characterised by angles of arrival $\left\{\vartheta_{l}, \varphi_{l}\right\}, l=1 \ldots L$, then using the broadband steering vector defined in (5) we obtain

$$
\boldsymbol{R}(z)=\sum_{l=1}^{L} \mathbf{s}\left(\vartheta_{l}, \varphi_{l}, z\right) \tilde{\mathbf{s}}\left(\vartheta_{l}, \varphi_{l}, z\right) S_{l}(z)+\sigma_{v}^{2} \mathbf{I}
$$

where $S_{l}(z)$ is the power spectral density of the $l$-th source measured at the origin, and $\sigma_{v}^{2}$ the power of the additive noise, whcih is assumed to be spatially and temporally uncorrelated.

\subsection{Minimum Variance Distortionless Response}

We denote $\boldsymbol{w}(z) \in \mathbb{C}^{M}$ to contain the complex conjugated and time-reversed beamforming filters, such that $\tilde{\mathbf{w}}(z) \bullet \mathrm{w}^{\mathrm{H}}[n]$ 


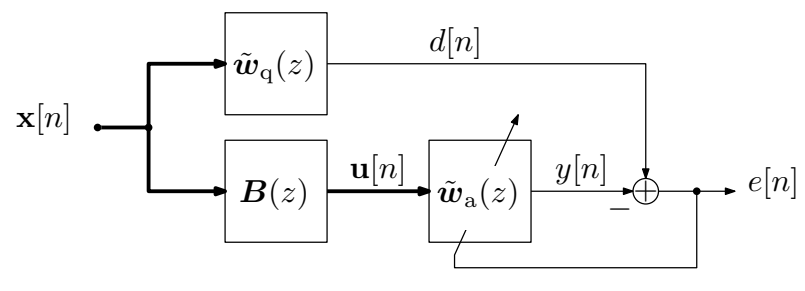

Figure 2. Generalised sidelobe canceller with polynomial quiescent vector $\boldsymbol{w}_{\mathrm{q}}(z)$, polynomial blocking matrix $\boldsymbol{B}(z)$ and a multichannel adaptive filter $\boldsymbol{w}_{\mathrm{a}}(z)$.

with $\mathbf{w}^{\mathrm{H}}[n]$ as defined in (7). Therefore, the output power spectral density of the beamformer is $\tilde{\boldsymbol{w}}(z) \boldsymbol{R}(z) \boldsymbol{w}(z)$ leads to the MVDR problem formulation

$$
\begin{array}{ll} 
& \min _{\mathbf{w}(z)} \oint_{|z|=1} \tilde{\boldsymbol{w}}(z) \boldsymbol{R}(z) \boldsymbol{w}(z) \frac{d z}{z} \\
\text { s.t. } & \tilde{\boldsymbol{s}}\left(\vartheta_{\mathrm{s}}, \varphi_{\mathrm{s}}, z\right) \boldsymbol{w}(z)=F(z) .
\end{array}
$$

The broadband steering vector $s\left(\vartheta_{\mathrm{s}}, \varphi_{\mathrm{s}}, z\right)$ protects the signal of interest emitted by a source with an elevation and azimuth of $\left\{\vartheta_{\mathrm{s}}, \varphi_{\mathrm{s}}\right\}$. In this look direction, the array is expected to provide a desired frequency response $F(z)$. For simplicity $F(z)=1$ will be assumed below.

\subsection{Generalised Sidelobe Canceller}

Instead of solving the broadband MVDR problem in (10) and (11) directly, the formulation has been extended to a GSC [23] as depicted in Fig. 2. This requires a quiescent beamformer $\mathbf{w}_{\mathrm{q}}$ which points in the look direction defined by $\left\{\vartheta_{\mathrm{s}}, \varphi_{\mathrm{s}}\right\}$ and minimises the output power spectral density $\tilde{\boldsymbol{w}}(z) \boldsymbol{R}(z) \boldsymbol{w}(z)$ for spatially white noise. The output of this quiescent beamformer, $d[n]=\mathbf{w}_{\mathrm{q}}^{\mathrm{H}} \mathbf{x}[n]$, generally still contains interference. By means of a blocking matrix $\mathbf{B}$, whose rows span the nullspace of $\mathbf{w}_{\mathrm{q}}$ such that $\mathbf{B} \mathbf{w}_{\mathrm{q}}=\underline{0}$, a signal $\mathbf{u}[n]=\mathbf{B} \mathbf{x}[n]$ is generated which contains only interference. Thereafter, unconstrained optimisation is applied to a noise cancelling filter $\mathbf{w}_{\mathrm{a}}$ such that the principle of orthogonality $\mathcal{E}\left\{\mathbf{u}[n] e^{*}[n]\right\}=\underline{0}$ is satisfied. The output $e[n]=d[n]-\mathbf{w}_{\mathrm{a}}^{\mathrm{H}} \mathbf{u}[n]$ then no longer contains interference.

\section{Implementation}

Below, some details are provided into how broadband steering vectors for (4) can be extracted, and how to obtain a blocking matrix $\boldsymbol{B}(z)$ from the constraint equation (11).

\subsection{Broadband Steering Vectors}

Fractional delay filters can be obtained by resampling the sinc function, which is the assumed interpolation function when sampling at Nyquist rate. Due to the infinite support of the sinc, a truncation is required, leading to a discrete prolate spheroidal sequence $[11,8]$. Other alternatives exist, such as Farrow filters [8]. In general, fractional delay filters cannot provide good accuracy towards $f_{\mathrm{s}} / 2$, but tapered windows for sinc functions [17] or the use of filter banks [1] can provide suitable implementations. We here use the proposition in [17] and apply a raised cosine or Hann window of length $2 L$ to the sinc, such that

$$
f[n-\tau]= \begin{cases}g[n] & -L+\tau<n<L+\tau, n \neq \tau \\ 2 & n=\tau \\ 0 & \text { otherwise }\end{cases}
$$

for (4) with

$$
g[n]=\frac{\sin \pi(n-\tau)}{\pi(n-\tau)}\left(1+\cos \frac{\pi}{L}(n-\tau)\right) .
$$

\subsection{Blocking Matrix}

The blocking matrix has to be designed such that

$$
\boldsymbol{B}(z) \boldsymbol{w}_{\mathrm{q}}(z)=\underline{0},
$$

which can therefore be achieved by completing $\boldsymbol{w}_{\mathrm{q}}(z)$ to a paraunitary matrix as suggested in [23]. This can be accomplished by applying a polynomial eigenvalue decomposition (PEVD, [10]) to the rank one matrix

$$
\boldsymbol{w}_{\mathrm{q}}(z) \tilde{\boldsymbol{w}}_{\mathrm{q}}(z)=\overline{\boldsymbol{Q}}(z) \boldsymbol{D}(z) \tilde{\overline{\boldsymbol{Q}}}(z)
$$

This PEVD is ordered and due to the definition of the steering vector, we have

$$
\boldsymbol{D}(z)=\operatorname{diag}\left\{\begin{array}{llll}
1 & 0 & \ldots & 0
\end{array}\right\}
$$

The paraunitary matrix $\overline{\boldsymbol{Q}}(z)$ is not unique even in the case where (15) has full rank. Defining the column vectors of the paraunitary matrix

$$
\overline{\boldsymbol{Q}}(z)=\left[\begin{array}{llll}
\overline{\boldsymbol{q}}_{1}(z) & \overline{\boldsymbol{q}}_{2}(z) & \ldots & \overline{\boldsymbol{q}}_{M}(z)
\end{array}\right],
$$

then e.g. $\overline{\boldsymbol{q}}_{1}(z)$ can be shifted w.r.t. $\boldsymbol{w}_{\mathrm{q}}(z)$,

$$
\overline{\boldsymbol{q}}_{1}(z)=z^{-\Delta} \boldsymbol{w}_{\mathrm{q}}(z),
$$

and yet satisfy both (15) and (16). Similarly, the remaining columns $\overline{\boldsymbol{q}}_{m}(z)$ can be arbitrarily shifted without affecting the validity of the decomposition. Therefore, if the blocking matrix

$$
\tilde{\boldsymbol{B}}(z)=\left[\begin{array}{lll}
\overline{\boldsymbol{q}}_{2}(z) & \ldots & \overline{\boldsymbol{q}}_{M}(z)
\end{array}\right]
$$

is selected, then $\tilde{\boldsymbol{B}}(z) \boldsymbol{w}_{\mathrm{q}}(z)=\underline{0}$ is guaranteed, but $\boldsymbol{B}(z)$ could have a larger order than necessary. By appropriately shifting rows and truncation of small leading and trailing coefficients of $\boldsymbol{B}(z)$ [3], it is possible to reduce this order.

\section{Simulations and Results}

Based on performance metrics in Sec. 5.1 and the definition of the beamforming scenario in Sec. 5.2, Sec. 5.3 provides the results for the unadapted, i.e. quiescent beamformer, while Sec. 5.4 highlights some details of the adaptation and the characteristics of the adapted beamformer. 
Table 1. Sensor locations for scenario 1 .

\begin{tabular}{|r||r|r|r|}
\hline$i$ & $x_{i}$ & $y_{i}$ & $z_{i}$ \\
\hline \hline 1 & -1.1242 & -1.8617 & 0.7471 \\
2 & -1.8118 & -1.7862 & 0.3559 \\
3 & 0.7155 & 0.1188 & 1.7217 \\
4 & 0.7172 & 0.6846 & 1.3847 \\
5 & 1.7388 & -1.9692 & 0.1077 \\
6 & -0.4660 & -0.4663 & -1.6321 \\
7 & 0.0777 & -1.7326 & 0.6157 \\
8 & 1.3239 & -0.3301 & -0.3360 \\
\hline
\end{tabular}

\subsection{Performance Metrics}

To assess the performance of broadband GSC beamformer, the mean square of the residual error $e_{\mathrm{r}}[n]$ is a useful metric. Since the beamformer output $e[n]$ should contain the signal of interest plus residual error, subtracting out the desired source signal - appropriately delayed to compensate for propagation delays in $\boldsymbol{w}_{\mathrm{q}}(z)$ - provides a metric similar to echo return loss enhancement known in acoustic echo cancellation.

The directivity pattern represents the gain response of a broadband beamformer w.r.t. the angles of arrival and frequency. Assuming that a broadband source is located at $\{\vartheta, \varphi\}$ as characterised by the broadband steering vector $s(\vartheta, \varphi, z)$, the overall transfer function of the beamformer w.r.t. this excitation is

$$
A(\vartheta, \varphi, z)=\left(\tilde{\boldsymbol{w}}_{\mathrm{q}}(z)-\tilde{\mathbf{w}}_{\mathrm{a}}(z) \boldsymbol{B}(z)\right) \cdot \boldsymbol{s}(\vartheta, \varphi, z) .
$$

By probing (20) with a series of steering vectors, the directivity pattern is obtained evaluating it on the unit circle and taking the gain only.

\subsection{Scenario}

An arbitary array with $M=8$ elements is generated by randomly drawing element locations from a uniform distribution for $\left\{x_{m}, y_{m}, z_{m}\right\} \in[-2 ; 2]$, i.e. all elements lie within a cube. The resulting coordinates are listed in Tab. 1.

The source of interest is set to illuminate the array from an elevation $\vartheta_{\mathrm{s}}=60^{\circ}$ and azimuth $\varphi_{\mathrm{s}}=-45^{\circ}$. A first interferer is located at $\left\{\vartheta_{\mathrm{i}, 1}=0^{\circ}, \varphi_{\mathrm{i}, 1}=-45^{\circ}\right\}$, with a second at $\left\{\vartheta_{\mathrm{i}, 2}=20^{\circ}, \varphi_{\mathrm{i}, 2}=90^{\circ}\right\}$. Both interferers have a signal-tointerference noise ratio of $-30 \mathrm{~dB}$, and additive white Gaussian noise at a level of $20 \mathrm{~dB}$ relative to the signal of interest. Both interferers have a highpass characteristic, with passband edge at $\Omega=\frac{\pi}{4}$.

\subsection{Quiescent Response}

The beampattern of the unadapted beamformer, i.e. with $\boldsymbol{w}_{\mathrm{a}}=$ $\underline{0}$, is the response of the quiescent beamformer only. The quiescent beamformer has been designed as a broadband steering vector consisting of fractional delay filters of order 40, which was found to provide sufficient accuracy.

Figs. 3 and 4 show the directivity patterns $\left|A\left(\vartheta, \varphi, e^{j \Omega}\right)\right|$ for two fixed points. Firstly, for $\vartheta=\vartheta_{\mathrm{s}}=60^{\circ}$, the beamformer is

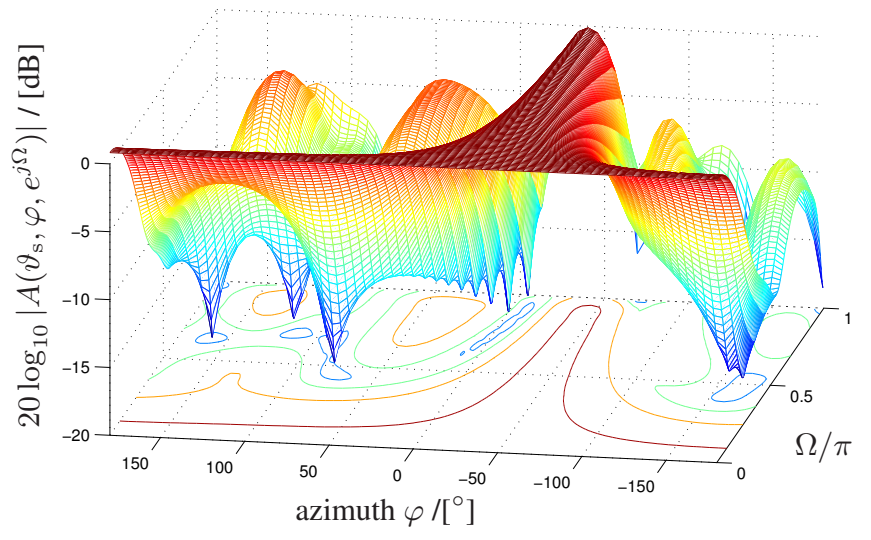

Figure 3. Directivity pattern of polynomial quiescent beamformer $\boldsymbol{w}_{\mathrm{q}}(z)$ in elevation look direction $\vartheta_{\mathrm{s}}=60^{\circ}$ for variable azimuth angle $\varphi$.

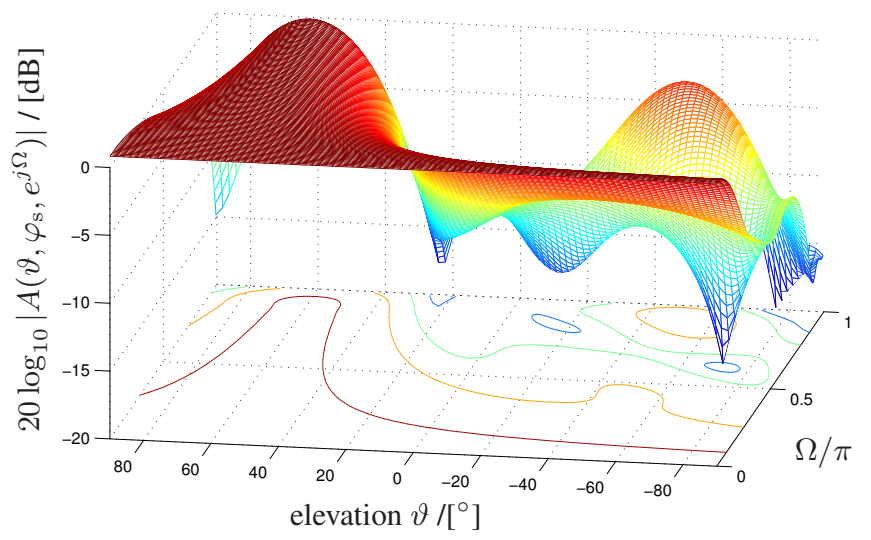

Figure 4. Directivity pattern of polynomial quiescent beamformer $\boldsymbol{w}_{\mathrm{q}}(z)$ in azimuth look direction $\varphi_{\mathrm{s}}=-45^{\circ}$ for variable elevation angle $\vartheta$.

pointing in the elevation direction of the source but with varying azimuth angle. Note that the gain response in Fig. 3 satisfies the constraint for $\varphi$, with a frequency-independent unit gain response in direction $\varphi=\varphi_{\mathrm{s}}=-45^{\circ}$. Secondly, fixing the azimuth angle to $\varphi=\varphi_{\mathrm{s}}=-45^{\circ}$ but varying the elevation $\vartheta$ in Fig. 4, the constraint is fulfilled for $\vartheta=\vartheta_{\mathrm{s}}=60^{\circ}$.

\subsection{Adapted Beamformer}

Based on the quiescent beamformer, a blocking matrix was designed by paraunitary matrix completion, resulting in a blocking matrix of order 480 after appropriate truncation [3]. The adaptive filter $\boldsymbol{w}_{\mathrm{a}}(z)$ was chosen of order 140 . Using a normalised LMS algorithm for unconstrained optimisation, the mean square residual error is depicted in Fig. 5.

Figs. 6 and 7 show the directivity patterns of the GSC beamformer after adaptation. In Fig. 6, the elevation angle is fixed at $\vartheta_{\mathrm{s}}=60^{\circ}$, such that the look direction of the array is observed for the flexible azimuth angle at $\varphi=\varphi_{\mathrm{s}}=-45^{\circ}$. The directivity pattern differs from the one of the quiescent beamformer in Fig. 3 but fulfils the constraint.

In Fig. 7, the adapted directivity pattern is shown for a fixed azimuth angle of $\varphi_{\mathrm{s}}=-45^{\circ}$. Compared to the quiescent response in Fig. 4, the constraint is still fulfilled but the beamformer has placed a null at the interference angle $\vartheta_{\mathrm{i}, 1}=0^{\circ}$. Since the interferer has a highpass characteristic, the loss of 


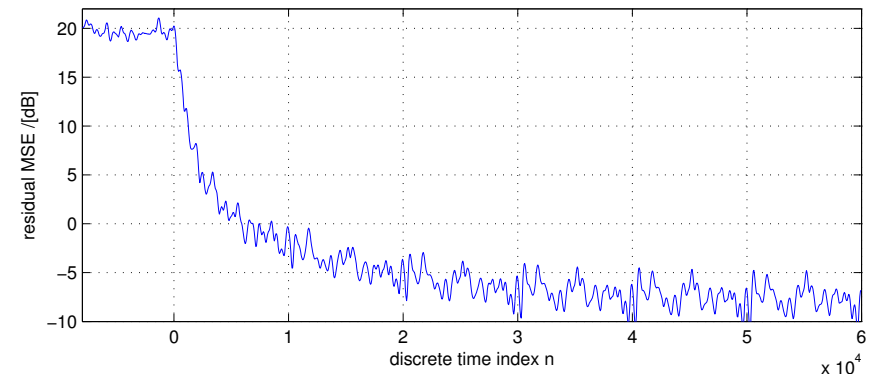

Figure 5. Mean square residual error after switching adaptation on at $n=0$.

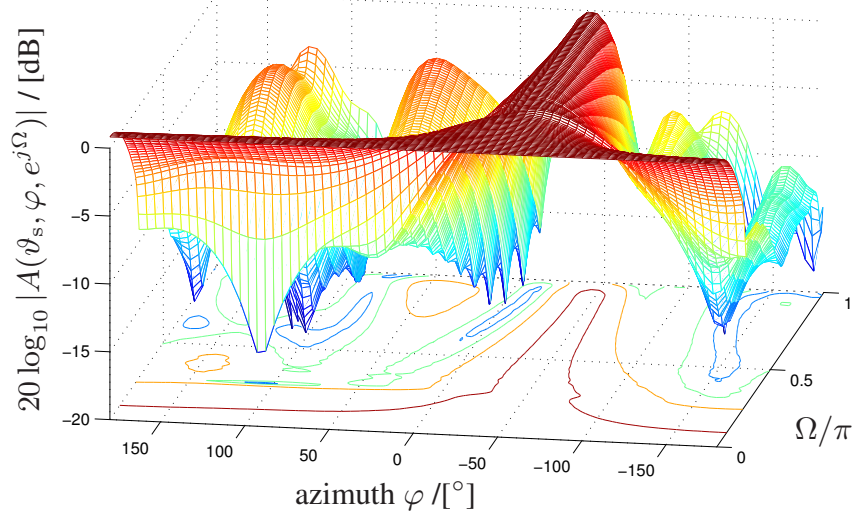

Figure 6. Directivity pattern of polynomial adapted beamformer $\boldsymbol{w}(z)$ in elevation look direction $\vartheta_{\mathrm{s}}=60^{\circ}$ for variable azimuth angle $\varphi$.

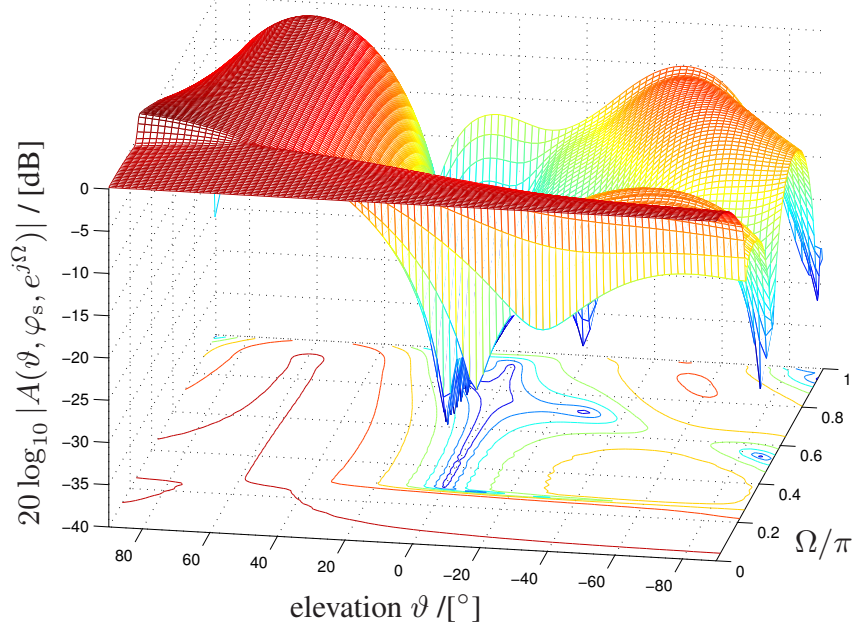

Figure 7. Directivity pattern of polynomial adapted beamformer $\boldsymbol{w}(z)$ in azimuth look direction $\varphi_{\mathrm{s}}=-45^{\circ}$ for variable elevation angle $\vartheta$.

spatial discrimination towards low frequencies by the beamformer has no impact on the beamformer's performance.

\section{Conclusion}

This paper has expanded on a proposed broadband beamformer design using polynomial matrix formulations. The design is derived using broadband steering vectors and a space-time covariance matrix that explicitly contains a lag parameter; the definition of the MVDR beamformer thus becomes a convenient extension of the narrowband case.
We have previously established some of the advantages of the proposed design - this includes the decoupling of orders in the quiescent beamformer, the blocking matrix, and the adaptive filter. Also, the proposed beamformer permits a simple handling of constraints. In the examples of this paper, we have shown that for an arbirary array configuration, constraints are straightforward to implement and are protected coherently across the spectrum. This is in contrast to state-of-the-art broadband beamformers, where off-broadside constraints or arbitrary array configurations generally require presteering before any processing can be performed.

\section{Acknowledgement}

This work was supported by the Engineering and Physical Sciences Research Council (EPSRC) Grant number EP/K014307/1 and the MOD University Defence Research Collaboration in Signal Processing.

\section{References}

[1] M. Alrmah and S. Weiss. Filter bank based fractional delay filter implementation for widely accurate broadband steering vectors. In 5th IEEE International Workshop on Computational Advances in Multi-Sensor Adaptive Processing, Saint Martin, December 2013.

[2] M. Alrmah, S. Weiss, and S. Lambotharan. An extension of the music algorithm to broadband scenarios using polynomial eigenvalue decomposition. In 19th European Signal Processing Conference, pages 629-633, Barcelona, Spain, August 2011.

[3] J. Corr, K. Thompson, S. Weiss, I. Proudler, and J. McWhirter. Row-shift corrected truncation of paraunitary matrices for PEVD algorithms. In 23rd European Signal Processing Conference, pages 854-858, Nice, France, August/September 2015.

[4] M. Davies, S. Lambotharan, and J. McWhirter. Broadband MIMO beamforming using spatial-temporal filters and polynomial matrix decomposition. In 15th International Conference on Digital Signal Processing, pages 579-582, Cardiff, UK, July 2007.

[5] L. Godara. Application of antenna arrays to mobile communications. II. Beam-forming and direction-of-arrival considerations. Proceedings of the IEEE, 85(8):1195-1245, Aug. 1997.

[6] I. Gohberg, P. Lancaster, and L. Rodman. Matrix Polynomials. Academic Press, New York, 1982.

[7] C. L. Koh, S. Redif, and S. Weiss. Broadband gsc beamformer with spatial and temporal decorrelation. In 17th European Signal Processing Conference, pages 889-893, Glasgow, Scotland, August 2009.

[8] T. I. Laakso, V. Välimäki, M. Karjalainen, and U. K. Laine. Splitting the Unit Delay. IEEE Signal Processing Magazine, 13(1):30-60, January 1996.

[9] W. Liu and S. Weiss. Wideband Beamforming - Concepts and Techniques. Wiley, 2010.

[10] J. G. McWhirter, P. D. Baxter, T. Cooper, S. Redif, and J. Foster. An EVD Algorithm for Para-Hermitian Polynomial Matrices. IEEE Transactions on Signal Processing, 55(5):21582169, May 2007.

[11] A. Papoulis. Signal Analysis. McGraw-Hill, New York, 1984.

[12] S. Redif, J. McWhirter, P. Baxter, and T. Cooper. Robust broadband adaptive beamforming via polynomial eigenvalues. In OCEANS, pages 1-6, Boston, MA, September 2006.

[13] S. Redif, J. McWhirter, and S. Weiss. Design of FIR paraunitary filter banks for subband coding using a polynomial eigenvalue decomposition. IEEE Transactions on Signal Processing, 59(11):5253-5264, November 2011. 
[14] S. Redif, S. Weiss, and J. McWhirter. Sequential matrix diagonalization algorithms for polynomial EVD of parahermitian matrices. IEEE Transactions on Signal Processing, 63(1):8189, January 2015.

[15] M. Rübsamen and A. Gershman. Robust presteered broadband beamforming based on worst-case performance optimization. In 5th IEEE Sensor Array and Multichannel Signal Processing Workshop, pages 340-344, Darmstadt, Germany, July 2008.

[16] M. Sayyah Jahromi and L. Godara. Steering broadband beamforming without pre-steering. In IEEE/ACES International Conference on Wireless Communications and Applied Computational Electromagnetics, pages 987-990, April 2005.

[17] J. Selva. An efficient structure for the design of variable fractional delay filters based on the windowing method. IEEE Transactions on Signal Processing, 56(8):3770-3775, August 2008.

[18] C. H. Ta and S. Weiss. A Design of Precoding and Equalisation for Broadband MIMO Systems. In 15th International Conference on Digital Signal Processing, pages 571-574, Cardiff, UK, July 2007.

[19] I. Thng, A. Cantoni, and Y. H. Leung. Derivative constrained optimum broad-band antenna arrays. IEEE Transactions on Signal Processing, 41(7):2376-2388, July 1993.

[20] P. P. Vaidyanathan. Multirate Systems and Filter Banks. Prentice Hall, Englewood Cliffs, 1993.

[21] B. D. Van Veen and K. M. Buckley. Beamforming: A Versatile Approach to Spatial Filtering. IEEE Acoustics, Speech, and Signal Processing Magazine, 5(2):4-24, April 1988.

[22] P. Vouras and T. Tran. Robust transmit nulling in wideband arrays. IEEE Transactions on Signal Processing, 62(14):37063719, July 2014

[23] S. Weiss, S. Bendoukha, A. Alzin, F. Coutts, I. Proudler, and J. Chambers. MVDR broadband beamforming using polynomial matrix techniques. In 23rd European Signal Processing Conference, pages 844-848, Nice, France, September 2015.

[24] S. Zhang and I.-J. Thng. Robust presteering derivative constraints for broadband antenna arrays. IEEE Transactions on Signal Processing, 50(1):1-10, January 2002. 